Dhaka Univ. J. Biol. Sci. 29(2): 219-227, 2020 (July)

\title{
ORCEIN AND FLUORESCENT BANDING ANALYSIS OF TWO FLORAL TYPES OF CATHARANTHUS ROSEUS L.
}

\author{
Tanusree Datta, Meghla Saha Pinky, Chandan Kumar Dash ${ }^{1}$ \\ AND KAZI NAHIDA BEGUM* \\ Department of Botany, Jagannath University, Dhaka-1100, Bangladesh
}

Key words: Fluorescent banding, Catharanthus roseus, Orcein, CMA, DAPI

\begin{abstract}
Two floral types of Catharanthus roseus L. viz. pink and white were studied through differential staining with orcein, CMA and DAPI for cytogenetical characterization and to assist towards updating their taxonomical status and evaluating chromosomal diversity between them. "Simple Chromocenter Type" of interphase nuclei was observed with some darkly stained small heterochromatic regions throughout the nuclei. Most of the prophase chromosomes of Catharanthus roseus (pink and white) were "Continuous Type" and a few were "Gradient Type". Although these two floral types possessed 16 metacentric chromosomes in somatic cells, they showed variation in fluorescent banding pattern considering the modification of GC- and AT-rich repetitive segments. Taking into account all the parameters of both the floral types of $C$. roseus showed strict symmetric karyotype as well as primitive nature. Therefore, the combined data of differential staining provide information to make comments on their chromosomal status with cytogenetical characterization and also create a baseline for future research.
\end{abstract}

\section{Introduction}

Catharanthus roseus L., (Synonyms: Vinca rosea L., Pervinca rosea L., Ammocallis rosea L. and Lochnera rosea L.) commonly known as periwinkle belongs to Apocynaceae is an evergreen, ever blooming, herbaceous and important medicinal plant as well as floral species in horticulture ${ }^{(1)}$. The word 'Catharanthus; derived from the Greek language meaning 'pure flower' while 'roseus' from Latin means red, rose or rosy(2).

Generally, two floral types of $C$. roseus are available which are named on the basis of their flower color i.e. with pink flower and reddish stems 'Rosea' and with white flower and green stems 'Alba', often found growing sympatrically(3). In Bangladesh, both floral types of C. roseus L. are available which is commonly known as 'Nayantara'.

*Author for correspondence: <kazinahida@yahoo.com>. ${ }^{1}$ Department of Botany, University of Dhaka, Dhaka-1000, Bangladesh. 
Catharanthus roseus is common in many tropical and subtropical regions throughout the world as a potential source of alkaloids ${ }^{(1)}$. This plant possesses many phytochemical constituents such as polyphenols, steroid, irridoid glucosides, flavonoid glucosides, carbohydrate, saponin and alkaloids. More than 400 alkaloids are present in different parts of this plant, which are used as pharmaceuticals, agrochemicals, flavor and fragrance, ingredients, food additives and pesticides. However, among all the alkaloids, only five consisting of vinblastine, vincristine, 3', 4'-anhydrovinblastine, serpentine and ajmalicine are marketed ${ }^{(4)}$. C. roseus, which is also known as "an anticancerous drug yielding plant" and this effectively treats diabetes, malaria, dengue fever, dysentery, insect bites, skin infection, diarrhea, leukemia, eye irritation, dyspepsia, toothache, sore throat and lung congestion ${ }^{(1,5,6)}$. Numerous researches have been initiated in order to increase the content of alkaloids and other biologically active constituents by producing new variants with enhanced bio-productivity. So, in this case authentic identification and genetical characterization is very important to carry out any breeding program.

These two floral types of $C$. roseus remain under taxonomic dilemma and also untouched for cytogenetical investigation in Bangladesh. Previous studies provided data mostly on chromosome counts of C. roseus which is not enough to provide detailed genomic information ${ }^{(7,8)}$. Karyosystematics is well established as important features in determination of the genetic relationship and degree of divergence among species or populations. Fluorescent chromosome banding is an excellent tool for karyotype analysis and provide information regarding the distribution of AT (Adenine - Thymine)- and GC (Guanine - Cytosine)-rich repeats in the genome ${ }^{(9)}$. Staining with DNA-base specific banding with fluorochromes such as chromomycin $\mathrm{A}_{3}$ (CMA) and 4', 6-diamidino-2phenylindole (DAPI) is relatively modern method for karyotype study. CMA binds with GC-rich repetitive sequences of the genome and gives characteristics yellow colour bands. On the other hand, DAPI binds with AT-rich repeats shows characteristic blue colour bands ${ }^{(9)}$. The nature of staining properties of interphase nuclei and prophase chromosomes are generally regarded as karyomorphological parameter. Tanaka (1971) classified different types of interphase nuclei and prophase chromosomes on the basis of heterochromatin condensation ${ }^{(10)}$. The combined karyomorphology and fluorescent banding analysis enable to characterize even different forms and varieties of a species. Therefore, the present study aimed at investigating different cytological characters to revise the taxonomic status and for characterization of two foral types of Catharanthus roseus available in Bangladesh.

\section{Materials and Methods}

The two floral types of Catharanthus roseus L. i.e. pink flower and white flower type were investigated. The specimens were collected from and maintained in the Botanical garden, Department of Botany, Jagannath University, Dhaka. 
The young healthy roots were collected and pretreated with $0.002 \mathrm{M}$ 8-hydroxyquinoline for $2 \mathrm{hrs} 30 \mathrm{~min}$ at room temperature followed by $15 \mathrm{~min}$ fixation in $45 \%$ acetic acid at $4^{\circ} \mathrm{C}$. These were then hydrolyzed in a mixture of $1 \mathrm{~N} \mathrm{HCl}$ and $45 \%$ acetic acid (3: 1) at $60^{\circ} \mathrm{C}$ for around $20 \mathrm{~min}$. The root tips were stained and squashed in $1 \%$ aceto-orcein. These were observed under a Nikon Eclipse 100. For CMA- and DAPI-banding, Alam and Kondo's(9) method was used with slight modification. After hydrolyzing and dissecting, the materials were tapped and squashed with $45 \%$ acetic acid and the cover glasses were removed quickly and allowed to air dry for at least $24 \mathrm{hrs}$ before study. The air-dried slides were first pre-incubated in McIlvaine's buffer $(\mathrm{pH} \mathrm{7.0)}$ for $30 \mathrm{~min}$ followed by distamycin A $(0.1 \mathrm{mg} / \mathrm{ml})$ treatment for $10 \mathrm{~min}$. The slides were rinsed mildly in Mcllvaine's buffer supplemented with $5 \mathrm{mM} \mathrm{MgSO}_{4}$ for $15 \mathrm{~min}$. One drop of CMA $(0.1 \mathrm{mg} / \mathrm{ml})$ was added and incubated for $1 \mathrm{hr}$ in a humid chamber and then rinsed with McIlvaine's buffer containing $5 \mathrm{mM} \mathrm{MgSO}_{4}$ for $10 \mathrm{~min}$. Slides were mounted in $50 \%$ glycerol and kept at $4^{\circ} \mathrm{C}$ for overnight before observation under Nikon (Eclipse 50i) fluorescent microscope with blue violet (BV) filter cassette. For DAPI-staining, after 24 hrs of air drying, the slides were first pre-incubated in Mcllvaine's buffer ( $\mathrm{pH} 7.0$ ) for 25 min and treated in actinomycin D $(0.25 \mathrm{mg} / \mathrm{ml})$ for $10 \mathrm{~min}$ in a humid chamber. The slides were immersed in DAPI solution $(0.01 \mathrm{mg} / \mathrm{ml})$ for $1 \mathrm{hr}$ and mounted with $50 \%$ glycerol and observed under a Nikon (Eclipse 50i) fluorescent microscope with UV filter cassette.

Centromeric formula was determined based on the position of centromere and the number of chromosomes ${ }^{(11)}$. Different karyomorphological parameters, asymmetry and symmetry indexes were calculated, such as range of chromosomal length, total chromosome length, average chromosome length, total form (TF\%) percentage ${ }^{(12)}$, karyotype asymmetry index (AsK\%)(13), karyotype symmetry index $(\mathrm{Syi} \%)^{(14)}$, degree of asymmetry of karyotypes (A)(15) and karyotypes category(16).

\section{Results and Discussion}

Although a few dividing cells were observed throughout the year, maximum number of dividing cells could be found in the root tip cells (RTCs) studied during May to August (about 60\%). The number of dividing cells was very poor in extreme high or low temperature.

Some darkly stained small heterochromatic regions were found with orcein-stained interphase nuclei of both pink and white floral types of $C$. roseus and 9 - 14 sharp CMA and DAPI band were observed under fluorescent banding. According to earlier reports, these features showed "Simple Chromocenter Type" of staining property in the interphase nuclei(9). Here, no nucleolus was observed in both types of $C$. roseus (Fig. 1).

The prophase chromosomes of the pink and white floral types were stained homogenously along the entire length i.e. "Continuous Type" and a few chromosomes 
showed "Gradient Type" where chromosomes were darker in one end and gradually faint to the other end. These might be due to the more aggregation of heterochromatins on those specific locations (Fig. 1). The findings indicated that the heterochromatins were aggregated in the interphase nuclei and then homogenously or gradually distributed in the prophase chromosomes.
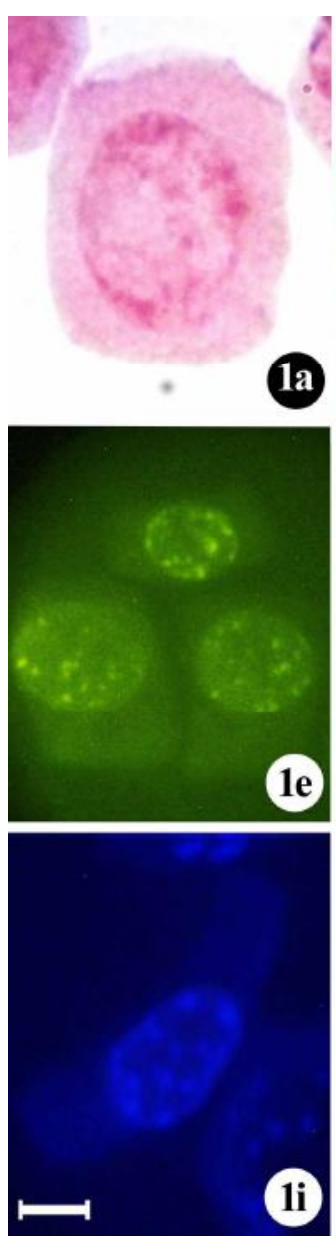
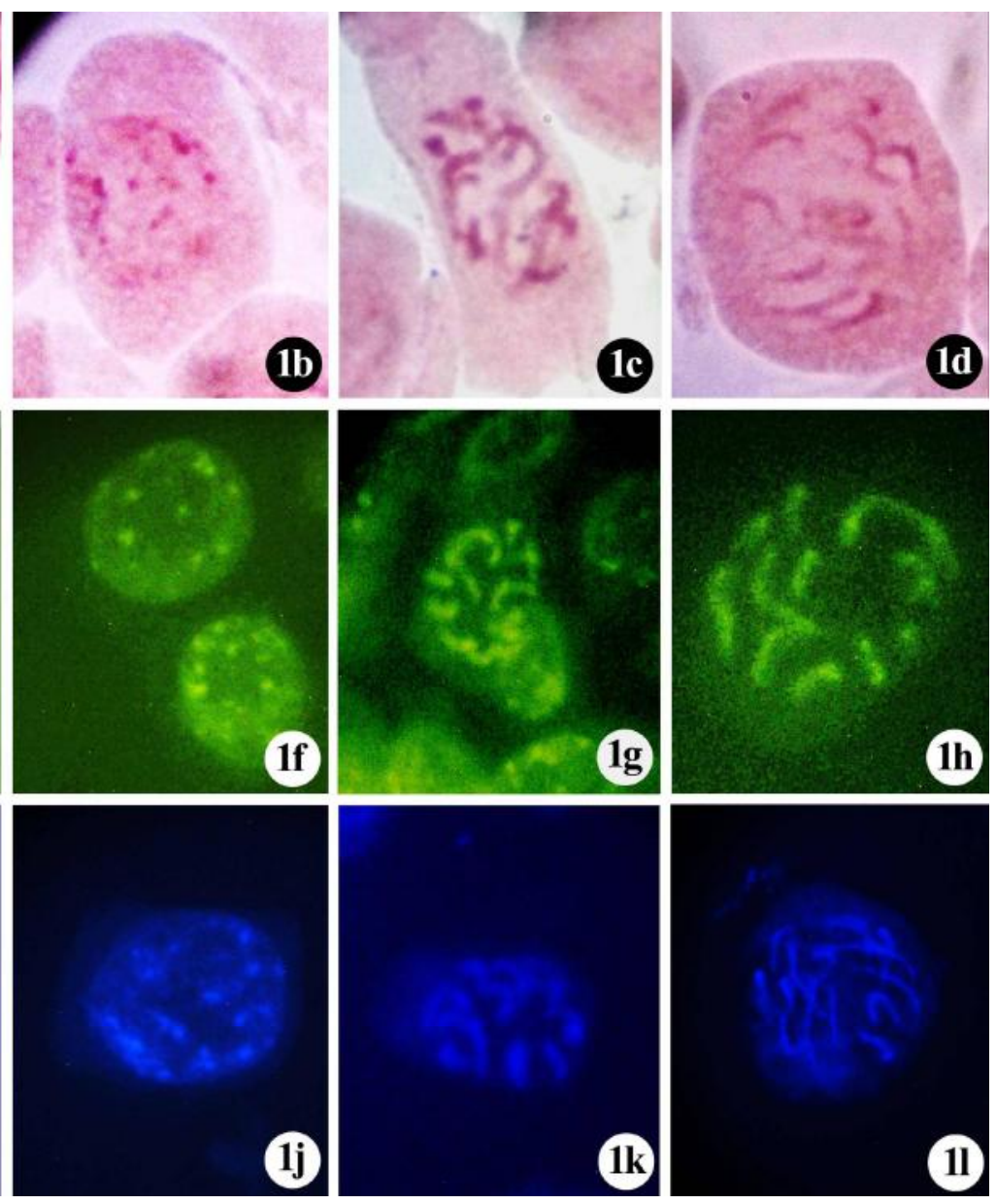

Fig. 1. Interphase nuclei and prophase chromosomes of two floral types. Orcein-stained interphase (1a) and prophase (1c), CMA-stained interphase (1e) and prophase (1g), DAPI-stained interphase (1i) and prophase (1k) of pink type. Orcein-stained interphase (1b) and prophase (1d), CMA-stained interphase (1f) and prophase (1h), DAPI-stained interphase (1j) and prophase (11) of white type. Bar $=10 \mu \mathrm{m}$.

According to the recorded chromosome number, different types or varieties of $C$. roseus are characterized by the basic chromosome number i.e., $x=8^{(17-19)}$. The diploid chromosome number $2 n=2 x=16$ was observed in both flower types of $C$. roseus in this investigation (Fig. 2). Similar diploid chromosome numbers were reported earlier which 
correlates to the present study $(7,8,20-23)$. The consistency in the number of diploid chromosome complement indicated that this species is stable. Considering basic chromo-

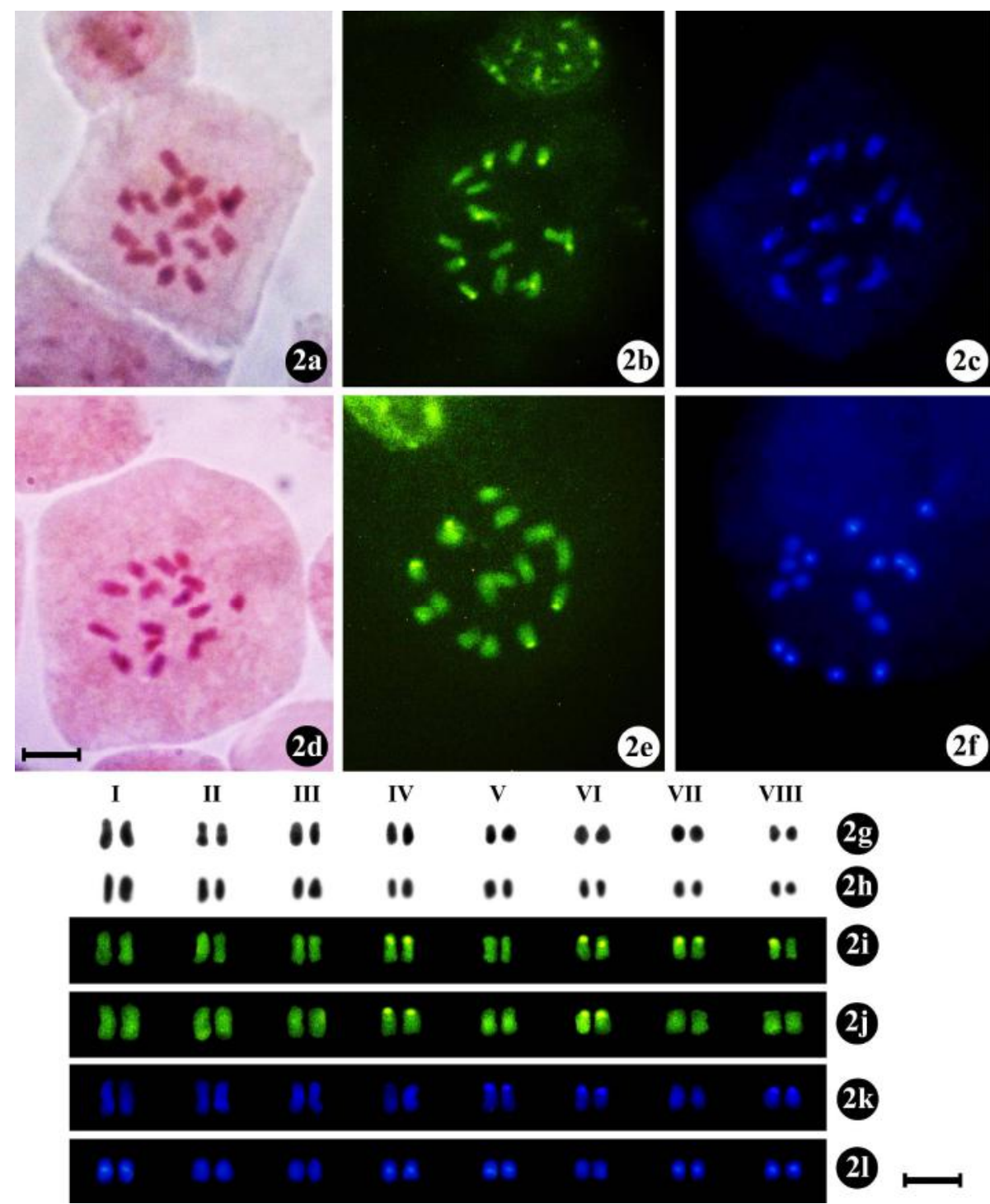

Fig. 2. Metaphase chromosomes and karyotypes of two floral types. Orcein-stained (2a, g), CMA-stained (2b, i) and DAPI-stained $(2 \mathrm{c}, \mathrm{k})$ metaphase and karyotype plates of pink type. Orcein-stained $(2 \mathrm{~d}, \mathrm{~h})$, CMA-stained $(2 \mathrm{e}, \mathrm{j})$ and DAPI-stained $(2 \mathrm{f}, 1)$ metaphase and karyotype plates of white type. Bars $=10 \mu \mathrm{m}$.

-some number $x=8$, the somatic chromosome number $2 n=24$ and 32 reported for this species might be triploid and tetraploid cytotype, respectively(20,24,25). Moreover, a few abnormal cells with $2 n=12$ and 14 chromosomes were also reported for this species, which did not correlate with the present findings ${ }^{(24)}$. Some previous workers reported the 
16 chromosomes of pink and white flower types with the different centromeric positions ${ }^{(21,22)}$. However, in the present study, 16 metacentric chromosomes were found in both pink and white flower type of C. roseus (Fig. 2). Karyotype having large number of metacentric chromosomes is usually regarded as symmetric type. Stebbins (1971) mentioned that symmetric karyotypes are of primitive features ${ }^{(16)}$. Therefore, both pink and white floral types of $C$. roseus possessed primitive karyotype in evolutionary point of view.

The total chromosomal and the average chromosomal length were found almost similar in pink and white floral types (43.39 and $2.71 \mu \mathrm{m} ; 43.54$ and $2.72 \mu \mathrm{m}$, respectively). The range of chromosomal length was $2.05-3.21 \mu \mathrm{m}$ in pink flower type and $2.26-3.42 \mu \mathrm{m}$ in white flower type (Table 1). Difference between small and large chromosome was more than about $1 \mu \mathrm{m}$ (Table 1).

Table 1. Differences in the karyomorphological parameters between the pink and white floral types.

\begin{tabular}{lll}
\hline Karyotype parameters & Pink type & White type \\
\hline Orcein-stained karyotype & & \\
Centromeric formula & $16 \mathrm{~m}$ & $16 \mathrm{~m}$ \\
Range of chromosomal length $(\mu \mathrm{m})$ & $2.05-3.21$ & $2.26-3.42$ \\
Total chromosome length $(\mu \mathrm{m})$ & $43.39 \pm 4.13$ & $43.54 \pm 0.89$ \\
Average chromosome length $(\mu \mathrm{m})$ & 2.71 & 2.72 \\
Total Form $(\mathrm{TF})$ value $(\%)$ & 47.80 & 48.51 \\
Karyotype asymmetry index $(\%)$ & 52.20 & 51.49 \\
Karyotype symmetry index $(\%)$ & 91.57 & 94.20 \\
Degree of asymmetry of karyotype $(\mathrm{A})$ & 0.05 & 0.03 \\
Karyotype category & $1 \mathrm{~A}$ & $1 \mathrm{~A}$ \\
CMA-stained karyotype & & \\
Number of CMA-band & 7 & 4 \\
Total CMA-banded portion & 6.49 & 3.79 \\
Percentage of CMA-banded portion $(\%)$ & 14.96 & 8.70 \\
DAPI-stained karyotype & & 10 \\
Number of DAPI-band & 6 & 6.54 \\
Total DAPI-banded portion & 4.90 & 15.02 \\
Percentage of DAPI-banded portion & 11.29 & \\
\hline
\end{tabular}

$\mathrm{m}=$ Metacentric chromosome.

Karyotype symmetry index (Syi\%) was somewhat lower in the pink flower type $(91.57 \%)$ than in the white flower type $(94.20 \%)$ of the plant. Whereas, karyotype asymmetry index $(\mathrm{AsK} \%)$ was little higher in pink flower type $(52.20 \%)$ than the white 
flower type (51.49\%) (Table 1). The total form percentage (TF) was more or less similar (47.80 for pink flower type and 48.51 for white flower type) for both types. The degree of karyotype asymmetry (A) is another parameter where the value was comparatively higher in pink flower type (0.05) than the white flower type (0.03). Karyotype category based on Stebbins's classification, "1A" karyotype was found for both pink and white floral types (Table 1) ${ }^{(16)}$. When the ratio of largest and smallest chromosome is less than $2: 1$, then it belongs to subtype ' $\mathrm{A}$ '(16). As pink and white flower types followed this ratio and both of them were categorized as "1A" degree of difference (Table 1).

After fluorescent banding, seven terminal CMA-bands were observed in pink floral type on the short arms of both chromosomes of pair IV, VI, VII and one chromosome of pair VIII. Possible heteromorphicity occurred in chromosome pair VIII where one chromosome had terminal band and its homologue did not show any band which might be due to the deletion of banded portion from the respective chromosome. The total CMA-banded portion was $6.49 \mu \mathrm{m}$ that occupied $14.96 \%$ of the total chromosome complements. In white floral type, four CMA-bands were found and those were observed at the terminal regions of the short arm of chromosome pair IV and VI. About $8.70 \%$ of the total somatic chromosome length was CMA-banded regions. In case of DAPI-banding, six and ten DAPI-bands were observed in pink and white floral types, respectively. In pink floral type, the DAPI-banded regions were at the terminal regions of the short arms (chromosome pair V, VI and VIII) while all the DAPI-bands were present at the centromeric regions in white floral type (chromosome pair I, IV, V, VII and VIII). The total length and percentage of the DAPI-banded regions were $4.90 \mu \mathrm{m}$ and 11.29 in pink floral type whereas these were $6.54 \mu \mathrm{m}$ and 15.02 in white floral type, respectively.

In spite of showing distinctiveness in fluorescent banding pattern, these two floral types had similar karyomorphology along with more or less similar total chromosome length and centromeric formula. Considering the above findings, it can be concluded that pink and white floral type possessed similar genome with some modification of banded regions (GC- and AT-rich repetitive segments). Therefore, the combined data of differential staining will be helpful for cytogenetical characterization and further investigation.

\section{Acknowledgement}

The financial support from the University Grants Commission, Government of the People's Republic of Bangladesh for carrying out this study is gratefully acknowledged.

\section{References}

1. Sain M and V Sharma 2013. Catharanthus roseus (An anti-cancerous drug yielding plant) are view of potential therapeutic properties. Int. J. Pure Appl. Biosci. 6: 139-142.

2. Kohlmuzer S 1968. Alkaloids of Catharanthus roseus (L.) G. Don - A new group of bio I active compounds. Postepy Biochem. 14(2): 209-232. 
3. Nejat N, A Valadiani, D Chachil, YH Tan, M Maziah and R Abiri 2015. Ornamental exterior versus therapeutic interior of madagascar periwinkle (Catharanthus roseus), the two faces of a versatile herb. Sci. World J. 2015: 1-19.

4. Hisiger S and M Jolicoeur 2007. Analysis of Catharanthus roseus alkaloids by HPLC. Phytochem. Rev. 6(2-3): 207-234.

5. Duke JA 1985. Handbook of medicinal herbs, Catharanthus roseus. Boca Raton, Fla, USA: CRC Press.

6. Sukumar K and Z Osmani 1981. Insect sterilants from Catharanthus roseus. Curr. Sci. 50(12): 55-58.

7. Tapadar $\mathrm{N}$ and $\mathrm{N}$ Roy 1964. Cytotaxonomic studies in Apocynaceae and delineation of the different evolutionary tendencies operating within the family. Caryologia 77: 103-138.

8. Mendioro MS, MG Diaz, MT Alcantara, OJ Hilario, P Mateo and DMR Maghirang 2005. Cytological studies of selected medicinal plants: Euphorbia pulcherrima Wild. ex Klotz., Moringa oleifera lam., Catharanthus roseus (L.) Don. and Chrysanthemum indicum Linn. Philipp. J. Sci. 134 (1): 31-37.

9. Alam SS and K Kondo 1995. Differential staining with orcein, giemsa, CMA and DAPI for comparative chromosome study of 12 species of Australian Drosera (Droseraceae). American J. Bot. 82: 1278-1286.

10. Tanaka R 1971. Type of resting nuclei in Orchidaceae. Bot. Mag. Tokyo 84: 118-122.

11. Levan A, K Fredga and AA Sandberg 1964. Nomenclature for centromeric position chromosome. Hereditas 52: 201-218.

12. Huziwara Y 1962. Karyotype analysis in some genera of Compositae. VIII. Further studies on the chromosomes of Aster. American J. Bot. 49: 116-119.

13. Arano H 1963. Cytological studies in subfamily Carduoideae (Compositae) of Japan IX. The karyotype analysis and phylogenetic considerations on Pertya and Ainsliaea. Bot. Mag. Tokyo 76: 32-39.

14. Greilhuber J and F Speta 1976. C-banded karyotypes in the Scilla hohenackeri group, S. persica and Puschkinia (Liliaceae). Plant Syst. Evol. 126: 149-188.

15. Watanabe K, T Yahara, T Denda and K Kosuge 1999. Chromosomal evolution in the genus Brachyscome (Asteraceae, Astereae): Statistical tests regarding correlation between changes in karyotype and habit using phylogenetic information. J. Plant Res. 112: 145-161.

16. Stebbins GL 1971. Chromosomal evolution in higher plants. Addison-Wesley publishing company, California, USA. pp. 208.

17. Furusatu K 1940. Quoted from Darlington CD and Janaki Ammal EK 1945, in chromosome atlas of cultivated plant, George Allen and Unwin, London.

18. Bowden WM 1945. A list of chromosome number in higher plants I, Acanthaceae to Myrtaceae. American J. Bot. 32: 81-92.

19. Rani N and K Kumar 2011. Karyomorphological studies in the genus Catharanthus. Indian J. Genet. 71(1): 55-60.

20. Dnyansagar VR and IV Sudhakaran 1970. Induced tetraploidy in Vinca rosea Linn. Cytologia 35: 227-241.

21. Kulkarni RN, K Baskaran and T Jhang 2016. Breeding medicinal plant, periwinkle, Catharanthus roseus (L.) G. Don: a review. Plant Genet. Resour. 1-20: 1479-2621. 
22. Jia CH, ZF Dai, BY Xu, ZQ Jin, L Zhang, YY Chen and JB Wang 2008. Analysis of karyotype of Catharanthus roseus (Apocynaceae). J. Trop.Subtrop. Bot. 16: 169-172.

23. De Padua DS, AA Barrion, CMV Casal and MPR De La Cruz 1992. Karyomorphology of chichirica Catharanthus roseus (L.) Don. Philipp. J. Sci. 121: 299-303.

24. Fedorov AA 1969. Chromosome numbers of flowering plants. Acad. Sci. USSR. pp. 48.

25. Datta PC and RK Maiti 1972. Relationships of Plumieraea (Apocynaceae) 1. Based on karyology. Bull. Bot. Soc. Bengal 26(1): 9-19.

(Manuscript received on 4 December, 2019; revised on 1 January, 2020) 